Mathematical Programming 10 (1976) 111-123.

North-Holland Publishing Company

\title{
PROJECTIONS IN MINIMAX ALGEBRA
}

\author{
R.A. CUNINGHAME-GREEN \\ Technische Hogeschool Twente, Enschede, The Netherlands
}

Received 3 February 1975

Revised manuscript received 22 April 1975

An axiomatic theory of linear operators can be constructed for abstract spaces defined over $(\mathbf{R}, \oplus, \otimes)$, that is over the (extended) real numbers $\mathbf{R}$ with the binary operations $x \oplus y=\max (x, y)$ and $x \otimes y=x+y$. Many of the features of conventional linear operator theory can be reproduced in this theory, although the proof techniques are quite different. Specialisation of the theory to spaces of $n$-tuples provides techniques for analysing a number of well-known operational research problems, whilst specialisation to function spaces provides a natural formal framework for certain familiar problems of approximation, optimisation and duality.

\section{A motivating example}

The object of the present article is to give an overview of a system of ideas which, because of the extensive nature of the research program of which they form a part (and the long refereeing procedures so implied), will not all be published in detailed form for some time. The accent in what follows is on explanation rather than on proof.

In the past 20 years (see references at the end) several different authors, often apparently unaware of one another's work, have discovered that an attractive formulation language is provided for a surprisingly wide class of problems by setting up an algebra of real numbers (perhaps extended by symbols such as $-\infty$, etc.) in which, however, the usual operations of multiplication and addition of two numbers are replaced by the operations:

(i) arithmetical addition, and

(ii) selection of the greater (or dually, the smaller) of the two numbers, respectively.

Thus, if $\mathbf{R}$ is the set of (suitably extended) real numbers, we define for 
each $x, y \in \mathbf{R}:$

$x \otimes y$ to be the arithmetical sum $x+y$,

$x \oplus y$ to be the quantity $\max (x, y)$ (or perhaps $\min (x, y))$.

A range of operational research problems (see e.g. [17]) including certain scheduling, shortest path, activity network, transportation, assignment, boolean, and queueing problems, when formulated using the notation (1.1), receive the form of problems of linear algebra. The techniques necessary to solve these problems, when generalised to function spaces, provide an intriguing formalism for approximation theory, optimisation and non-linear duality.

To illustrate such a diversity of matters in the compass of one article, it is necessary to single out one topic in which the main ideas all play some role. Accordingly, we shall content ourselves with describing an analogue of the classical projection theorem which we have proved, whose function-space generalisation gives a formal framework for (inter alia) the Fenchel duality theory. This new material is presented in Section 3 et sequ. But first, a simple motivating example, chosen from one of the several application fields.

A common feature of industrial processes is that machines do not act independently, and a typical machine cannot begin a fresh cycle of activity until certain other machines have all completed their current cycles.

A natural way of describing such a system is to label the machines e.g. $1, \ldots, n$, and to describe the interferences by recurrence relations such as:

$$
x_{3}(r+1)=\max \left(x_{1}(r)+t_{1}(r), \quad x_{2}(r)+t_{2}(r)\right) .
$$

This expresses the fact that machine 3 must wait to begin its $(r+1)^{\text {st }}$ cycle until machines 1 and 2 have both finished their $r^{\text {th }}$ cycle, the symbol $x_{i}(r)$ denoting the starting time of the $r^{\text {th }}$ cycle of machine $i$, and the symbol $t_{i}(r)$ denoting the corresponding activity duration.

This mode of analysis gives rise to formidable-looking systems of recurrence relations:

$$
x_{i}(r+1)=\max \left(x_{1}(r)+b_{i 1}, \ldots, x_{n}(r)+b_{i n}\right), \quad i=1, \ldots, n
$$

where, for notational uniformity, all terms $b_{i j}$ and $x_{j}(r)$, for $j=1, \ldots, n$, are made to occur for each $i$ by introducing where necessary quantities $b_{i j}$ equal to "minus infinity" for each combination $(i, j)$ which has no 
physical significance; the operator $\max$ will then "ignore" these terms.

Let us now make a change of notation. Write:

$$
\begin{aligned}
& x \oplus y \text { instead of } \max (x, y), \\
& x \otimes y \quad \text { instead of } x+y .
\end{aligned}
$$

Expression (1.2) becomes:

$$
x_{i}(r+1)=\left(b_{i 1} \otimes x_{1}(r)\right) \oplus \ldots \oplus\left(b_{i n} \otimes x_{n}(r)\right), \quad i=1, \ldots, n
$$

which is a kind of inner product. Introduce the obvious vector-matrix notation

$$
B=\left[b_{i j}\right], \quad x(r)=\left[x_{j}(r)\right]
$$

and (1.4) becomes:

$$
x(r+1)=B \otimes x(r) .
$$

Relation (1.5) enables a number of operational questions to be given a linear-algebra format. For example, to the operational question, "How must the system be set in motion so as to ensure that, for some given $r$, the $r^{\text {th }}$ cycles are undertaken at preassigned times?" corresponds the "simultaneous linear equations" problem [4]:

$$
\text { Solve } A \otimes x=b \quad \text { (where } A=B \otimes B \otimes \ldots \otimes B=B^{r} \text { ). }
$$

This problem does not in general have a solution, and in practice it is usually replaced by some problem with a criterion of best-approximation - for example by the minimax earliness problem (see also [8]):

$$
\begin{array}{ll}
\text { minimise } & \max _{1 \leqslant i \leqslant n}\left(b_{i}-(A \otimes x)_{i}\right), \\
\text { subject to } & A \otimes x \leqslant b .
\end{array}
$$

The eigenvector-value problem $B \otimes x=\lambda \otimes x$ has a physical interpretation, too [7]; it concerns the ability of the system to proceed in regular steps. The convergence of the series $\left\{\boldsymbol{B}^{r}\right\}(r=1,2, \ldots)$ then determines whether the system will seek this stable mode of behaviour.

Other examples of formalisms of this kind, arising in the application fields cited above, can be found in the references in Section 8 .

\section{Basic algebraic properties}

With the notation (1.3), the system $(\mathbf{R}, \oplus, \otimes)$ forms a commutative group under the operation $\otimes$ (a partial semigroup if infinite elements oc- 
cur) and a semilattice under the operation $\oplus$, i.e., for all $x, y, z \in \mathbf{R}$ there hold: $x \oplus x=x ; x \oplus(y \oplus z)=(x \oplus y) \oplus z ; x \oplus y=y \oplus x$.

Moreover, the distributive laws hold:

$$
\begin{aligned}
& (y \oplus z) \otimes x=(y \otimes x) \oplus(z \otimes x), \\
& x \otimes(y \oplus z)=(x \otimes y) \oplus(x \otimes z) .
\end{aligned}
$$

Algebraically, $(\mathbf{R}, \oplus, \otimes)$ can be viewed as a semilattice-ordered group or (partial) semigroup; or as a special kind of semiring in which all elements are additively idempotent.

Using the system $(\mathbf{R}, \oplus, \otimes)$ as scalars, we may define the analogue of a vector space $V$ by the properties $u \oplus v \in V$ and $\lambda \otimes u \in V$ for all $u, v \in V$ and $\lambda \in \mathbf{R}$. The research program alluded to above is concerned with developing a systematic theory of "linear operators" for such spaces, i.e. operators $A: U \rightarrow V$ such that

$$
\begin{aligned}
& \forall(\lambda, \mu \in \mathrm{R} ; u, v \in V) A[(\lambda \otimes u) \oplus(\mu \otimes v)]= \\
& \quad=[\lambda \otimes A(u)] \oplus[\mu \otimes A(v)] .
\end{aligned}
$$

In the first stage of the program, the argument was restricted to spaces of $n$-tuples, for which the linear operators are matrices. If $m_{m n}$ represents the set of $(m \times n)$ matrices over $\mathbf{R}$, then we define

$$
\begin{array}{r}
{\left[a_{i j}\right] \oplus\left[b_{i j}\right]=\left[a_{i j} \oplus b_{i j}\right] \quad \text { for }\left[a_{i j}\right],\left[b_{i j}\right] \in \mathcal{M}_{m n} ;} \\
{\left[a_{i j}\right] \otimes\left[b_{i j}\right]=\left[\sum_{r=1}^{p}\left(a_{i r} \otimes b_{r j}\right)\right] \quad \text { for }\left[a_{i j}\right] \in m_{m p},} \\
{\left[b_{i j}\right] \in m_{p n},}
\end{array}
$$

where the notation $\Sigma_{\oplus}$ denotes iterated use of the (commutative associative) operation $\oplus$.

The system $\mathbb{M}_{n n}$ is a non-commutative (partial) semigroup under the operation $\otimes$ and a semilattice under the operation $\oplus$, and the distributive laws (2.1) hold. The algebraic operations in $m_{n n}$ are also all isotone: for example if $A, B, C \in \mathcal{M}_{n n}$ and $B \geqslant C$ (in the sense that $b_{i j} \geqslant c_{i j}$ for all $(i, j))$, then $A \otimes B \geqslant A \otimes C$.

The beginnings of such a theory of matrix algebra have existed for some 20 years, but our aim has been to develop a comprehensive theory, with new material related to the eigenvector-value problem, projections, 
the spectral theorem, subspaces, approximation, norms, canonical forms, and an extensive duality theory. These results, which have useful interpretations in a number of application fields, were presented at the 10th Netherlands Mathematics Congress in April 1974, and are now being prepared for publication.

In the second stage of the program, the analysis is being extended to function spaces. The present note aims to give some of the flavour of the total investigation, and the material which now follows is, to the best of our knowledge, new.

\section{The conjugate}

The solution of problems (1.6) and (1.7) requires the development of a duality. Alongside the addition operation $\oplus$ we must introduce the dual addition $\oplus^{\prime}$ with the interpretation $x \oplus^{\prime} y=\min (x, y)$. The scalars $\mathbf{R}$ then enjoy three algebraic operations $\left(\oplus, \otimes, \oplus^{\prime}\right)$ but the matrices also have a dual multiplication $\otimes^{\prime}$ defined by using $\oplus^{\prime}$ in place of $\oplus$ in (2.3). For the algebraically rich system $\left(\mathcal{M}_{n n}, \oplus, \otimes, \oplus^{\prime} ; \otimes^{\prime}\right.$ ) (a sort of double lattice-ordered semigroup) it is possible to prove a large number of interesting inequalities and identities, but we shall restrict our attention now to the following.

Firstly, for $A=\left[a_{i j}\right] \in m_{m n}$ we define the conjugate of $A$ to be $A^{*}=\left[-a_{j i}\right] \in m_{n m}$, i.e., $A^{*}$ is derived from $A$ by transposing and negating. This definition of course extends to vectors ( $n$-tuples), if we regard them as $(n \times 1)$ matrices.

It is easy to see that $\left(A^{*}\right)^{*}=A ;(A \oplus B)^{*}=A^{*} \oplus^{\prime} B^{*} ;(A \otimes B)^{*}=$ $B^{*} \otimes^{\prime} A^{*}$ etc. And the following identities and inequalities hold for arbitrary matrices, provided they are conformable for the relevant products:

$$
\begin{aligned}
& A \otimes\left(A^{*} \otimes^{\prime} A\right)=A \otimes^{\prime}\left(A^{*} \otimes A\right)=\left(A \otimes^{\prime} A^{*}\right) \otimes A \\
& =\left(A \otimes A^{*}\right) \otimes^{\prime} A=A, \\
& A^{*} \otimes\left(A \otimes^{\prime} A^{*}\right)=A^{*} \otimes^{\prime}\left(A \otimes A^{*}\right)=\left(A^{*} \otimes^{\prime} A\right) \otimes A^{*} \\
& =\left(A^{*} \otimes A\right) \otimes^{\prime} A^{*}=A^{*}, \\
& A \otimes\left(A^{*} \otimes^{\prime} B\right) \leqslant B \leqslant A \otimes^{\prime}\left(A^{*} \otimes B\right), \\
& A \otimes\left(A^{*} \otimes^{\prime}(A \otimes X)\right)=A \otimes X .
\end{aligned}
$$


This is merely a selection, relevant to the present problem, from the available identities. All obvious duals, left-right associates and higherorder generalisations are also valid. Because of the presence of infinite elements, the proofs are a little delicate and the results hold under a small restriction of generality which there is no space to discuss here.

The resemblance of $A^{*}$ to a pseudo-inverse is striking ((3.1), (3.2)), and indeed $m_{n n}$ has some properties analogous to those of a regular ring [25] in the sense of $v$. Neumann, but with the extra complication of two different multiplication operations. The resemblance is even more striking in the following:

Theorem 1. Problem (1.6) has a solution if and only if $\boldsymbol{x}=\boldsymbol{A}^{*} \otimes^{\prime} \boldsymbol{b}$ is a solution; and then $x=A^{*} \otimes^{\prime} b$ is actually the greatest solution.

Proof. The "if" is trivial. So suppose $\boldsymbol{u}$ exists such that $\boldsymbol{A} \otimes \boldsymbol{u}=\boldsymbol{b}$. Then using (3.4),

$$
A \otimes\left(A^{*} \otimes^{\prime} b\right)=A \otimes\left(A^{*} \otimes^{\prime}(A \otimes u)\right)=A \otimes u=b .
$$

Hence $x=A^{*} \otimes^{\prime} b$ is also a solution. Moreover, from (3.3) with $A^{*}$ written for $A$,

$$
u \leqslant A^{*} \otimes^{\prime}(A \otimes u)=A^{*} \otimes^{\prime} b=x .
$$

Theorem 2. Problem (1.7) has the solution $\boldsymbol{x}=\boldsymbol{A}^{*} \otimes^{\prime} \boldsymbol{b}$; moreover this is the greatest solution.

Proof. From (3.3): $A \otimes x=A \otimes\left(A^{*} \otimes^{\prime} b\right) \leqslant b$. Hence $x$ is feasible for problem (1.7). Moreover if $u$ is such that $A \otimes u \leqslant b$, then, because matrix multiplication is isotone, we have, using (3.3) with $A^{*}$ written for $A$,

$$
u \leqslant A^{*} \otimes^{\prime}(A \otimes u) \leqslant A^{*} \otimes^{\prime} b=x .
$$

Hence $\boldsymbol{x}$ is the greatest feasible solution for problem (1.7). Also, by isotonicity,

$$
A \otimes u \leqslant A \otimes x .
$$

Hence $x$ is the desired minimax solution for problem (1.7).

\section{Projections}

The material of Section 3 generates a number of interesting thoughtassociations from different branches of mathematics. We have already 
remarked on the pseudo-inverse property of $A^{*}$. We now see that the set of $n$-tuples $u$ satisfying $A \otimes u \leqslant b$ has a greatest member $x\left(=A^{*} \otimes^{\prime} b\right)$, that is to say that we are concerned with a residuated algebraic structure. Furthermore from (3.3) and (3.4), the mapping

$$
\theta: B \rightarrow A \otimes^{\prime}\left(A^{*} \otimes B\right)
$$

is a closure operation in that $\boldsymbol{B} \leqslant \theta(\boldsymbol{B})=\theta(\theta(\boldsymbol{B}))$. The related mappings

$$
\sigma: C \rightarrow C^{*} \otimes^{\prime} A \text { and } \psi: D \rightarrow A \otimes^{\prime} D^{*}
$$

jointly define a Galois connection and $\theta$ of (4.1) can be written $\theta=\psi \sigma$. (see e.g. [18 and 19] in relation to these concepts). Furthermore:

Theorem 3. The mappings

$$
\alpha: \boldsymbol{y} \rightarrow A^{*} \otimes^{\prime} \boldsymbol{y} \quad \text { and } \beta: x \rightarrow A \otimes \boldsymbol{x}
$$

are mutually inverse $(1,1)$ mappings of the column-spaces $S=\{\boldsymbol{A} \otimes \boldsymbol{x}\}$ and $T=\left\{A^{*} \otimes^{\prime} y\right\}$ onto one another.

Proof. $\beta$ is onto because, by (3.4),

$$
A \otimes x=\beta\left(A^{*} \otimes^{\prime}(A \otimes x)\right) \in \beta(T)
$$

and similarly, $\alpha$ is onto. Moreover, by (3.4),

$$
\beta(\alpha(A \otimes x))=A \otimes x .
$$

Hence $\beta \alpha$ acts as the identity mapping on $S$ and similarly $\alpha \beta$ acts as the identity mapping on $T$.

For the present readership, we can look at these purely algebraic results in yet another way - i.e., as the solution to a minimum-norm problem. Problem (1.7) is concerned with finding a minimax-deviation underestimate $\boldsymbol{A} \otimes \boldsymbol{x}$ of $b$, that is to say, a best underestimating approximation $A \otimes x$ for $b$, in the Tchebychev norm.

According to Theorem 2, the maximal solution to this problem is: $A \otimes\left(A^{*} \otimes^{\prime} b\right)$. It is therefore natural to call the mapping $\phi: b \rightarrow A \otimes$ $\left(A^{*} \otimes^{\prime} b\right)$ the projection of $b$ on the column-space of $A$. With this definition it is possible to introduce projection matrices $P(\xi)$ such that $P(\xi) \otimes^{\prime} x$ is the projection of arbitrary $n$-tuple $x$ on $n$-tuple $\xi$. Such projection matrices have the usual formal properties (i.e., they are idempotent and self-conjugate and a given matrix $A$ has a spectral resolution as 
$\mathrm{a} \oplus^{\prime}$-sum of the projection matrices associated with its eigenvectors). The similarity to the classical case is thus extensive although the proof techniques are quite different because of the lack of an additive inverse operation for scalars and matrices. Further details will be given in future publications.

\section{Extension to function spaces}

The entire theory as so far discussed for $n$-tuples extends without difficulty to other more general spaces. To avoid too much abstraction, we consider here merely the generalisation for which $n$-tuples are replaced by real-valued functions $f(\cdot), g(\cdot)$ etc. of several real variables, and matrices $A=\left[a_{i j}\right]$ are replaced by real-valued functions $A(\cdot, \cdot)$ of two sets of several real variables. Matrix multiplication (2.3) is replaced by its formal analogue:

$$
\begin{aligned}
(A \otimes B)(x, y) & =\max _{z}\{A(x, z)+B(z, y)\} \\
& =\sum_{z}\{A(x, z) \otimes B(z, y)\} .
\end{aligned}
$$

Thus, equation (5.1) defines the function $A \otimes B$ of two sets of variables, given the functions $A(\cdot, \cdot)$ and $B(\cdot, \cdot)$. More generally, the maximisation in (5.1) would be over some domain of values of $z$, and the operator $\max$ would be replaced by the operator sup. For present simplicity of exposition, however, we use (5.1).

We may now define the dual product $A \otimes^{\prime} B$ by using min in place of max in (5.1) and the conjugate of $A(\cdot, \cdot)$ is $A^{*}(\cdot, \cdot)$ such that

$$
A^{*}(x, y)=-A(y, x) \text {. }
$$

With these definitions, pretty well the whole theory goes over from the space of $n$-tuples to the space of functions, including the eigenproblem, the spectral theorem, and so on, and provides interesting sidelights on the Stone-Weierstrass theorem, the Banach fixpoint theorem, the theory of convexity and nonlinear duality, and other related matters. In the present note, we have space only to sketch how the material of Sections 3 and 4 generalises.

Firstly, the identities and inequalities of Section 3 can readily be developed, under suitable conditions of semi-continuity and compactness. 
Now let us say that a function $f(\cdot)$ lies in the column-space of $A(\cdot, \cdot)$ if and only if we can find a function $g(\cdot)$ such that

$$
A \otimes g=f .
$$

The reasoning of Theorem 1 can be applied verbatim to show that this problem is soluble if and only if $g=A^{*} \otimes^{\prime} f$ is a solution, and then $g$ is the greatest solution in that every other solution $h$ satisfies $h(x) \leqslant g(x)$ identically, where

$$
\begin{aligned}
g(x)=\left(A^{*} \otimes^{\prime} f\right)(x) & =\min _{y}\left\{A^{*}(x, y)+f(y)\right\} \\
& =\min _{y}\{-A(y, x)+f(y)\} .
\end{aligned}
$$

If problem (5.3) does not have a solution, the reasoning of Theorem 2 can be applied verbatim to show that the function $A \otimes\left(A^{*} \otimes^{\prime} f\right)$ is a minimax-deviation underestimate for the function $f$, lying in the columnspace of $A$, and is indeed the greatest such minimax-deviation underestimate. Otherwise expressed, $A \otimes\left(A^{*} \otimes^{\prime} f\right)$ is the greatest best-approximating support function for $f$ lying in the column-space of $A$, relative to the Tchebychev norm, and the mapping

$$
\phi: f \rightarrow A \otimes\left(A^{*} \otimes^{\prime} f\right)
$$

acts as the projection of $f$ on the column-space of $A$, and has the appropriate formal properties of a projection.

\section{Convex functions}

The column-space of a given $A(\cdot, \cdot)$ characterises a class of functions. For example:

Theorem 4. The function $f$ is convex if and only if it lies in the columnspace of the inner product function $Q(\cdot, \cdot)$, where $Q(x, y)=\langle x, y\rangle$, the real inner product.

Proof. On the one hand, if $f=A \otimes g$, i.e., if

$$
f(x)=\max _{y}\{A(x, y)+g(y)\},
$$

then $f$ satisfies Jensen's inequality if $A$ is convex in $x$, in particular if $A$ is linear in $\boldsymbol{x}$, as $Q$ indeed is. 
On the other hand, if $f$ is convex, then it has a subgradient, i.e.,

$$
\forall x \exists u(x) \forall y f(y)-f(x)-\langle(y-x), u\rangle \geqslant 0 .
$$

It is not difficult to show that this implies:

$$
\forall x \max _{u} \min _{\boldsymbol{y}}\{f(y)-f(x)-\langle(y-x), u\rangle\}=0 .
$$

Rewriting,

$$
\forall x f(x)=\max _{u}\left\{\langle x, u\rangle+\min _{\boldsymbol{y}}\{-\langle y, u\rangle+f(y)\}\right\} .
$$

But this is: $f=Q \otimes\left(Q^{*} \otimes^{\prime} f\right)$.

An evident dual result applies to concave functions. The relationship to the Fenchel duality theory [28] is now transparent: indeed the mapping

$$
\psi: g \rightarrow Q \otimes^{\prime} g^{*}
$$

produces exactly the concave conjugate function of $g$, as defined by Fenchel (who defined it for concave functions only). The mapping $\psi$ (as observed in Section 4 above) forms part of a Galois connection. Fenchel's convex conjugate of a convex function $f$ is produced by the mapping

$$
\tau: f \rightarrow Q \otimes f^{*}
$$

and is clearly convex because it is of the form $Q \otimes \mathrm{g}$.

Fenchel gave separate definitions of the conjugate for convex and concave functions, but this formal asymmetry disappears if we merely consider the mappings

$$
\alpha: f \rightarrow Q^{*} \otimes^{\prime} f \text { and } \beta: g \rightarrow Q \otimes g
$$

which map "any" function into a concave, and into a convex, function respectively.

From Theorems 3 and 4 we see that $\alpha$ and $\beta$ are mutually inverse $(1,1)$ mappings of the classes of convex and of concave functions onto one another. Furthermore the projection $\phi=\beta \alpha$,

$$
\phi: f \rightarrow Q \otimes\left(Q^{*} \otimes^{\prime} f\right)
$$

has, using the discussion in Section 4, the property that it takes a given function $f$ into a convex function of support which best approximates 
$f$ in the Tchebychev norm, and a dual statement is true of the projection $\alpha \beta$. The function $\phi(f)$ is discussed (in other terms) in [22], where it is called the convex envelope of $f$, and it provides a way of turning a general minimisation problem into a convex problem, since $f$ and its convex evelope have the same minimum point and value.

Finally it is clear from Sections 5 and 6 that the Fenchel duality is just one particular case of a more general range of possibilities with $Q$ replaced by $A$.

\section{Conclusion}

In this communication, we have drawn attention to the fact that an axiomatic theory of linear operators can be constructed for abstract spaces defined over $(\mathbf{R}, \oplus, \otimes)$, which is rich enough to reproduce most of the features of conventional linear operator theory (although the proof techniques are quite different), and whose specialisations to e.g. the space of $n$-tuples or the space of real functions provide techniques of formulation and analysis for a number of problems having some importance in contemporary applied mathematics.

Optimisation techniques based upon the differential calculus and its generalisations have a number of weaknesses, such as failure to distinguish global from local optima. One of the motives behind the present research program is the idea that a theory in which the operators $\max$ and $\min$ enter at the axiomatic level may avoid some of these difficulties. The name minimax algebra is suggested for this theory.

\section{Chronological reference list on minimax algebra}

[1] A. Shimbel, "Structure in communication nets", Proceedings of the symposium on information networks, Polytechnic Institute of Brooklyn (1954) pp. 199-203.

[2] F.E. Hohn, S. Seshu and D.D. Aufenkamp, "The theory of nets", Transactions of the Institute of Radio Engineers EC-6 (1957) 154-161.

[3] B. Roy, "Transitivité et connexité", Comptes Rendus Hebdomadaires des Séances de l'Académie des Sciences de Paris 249 (1959) 216-218.

[4] R.A. Cuninghame-Green, "Process synchronisation in a steelworks - a problem of feasibility", in: Banbury and Maitland, ed., Proceedings of the 2 nd international conference on operational research (English University Press, 1960) pp. 323-328.

[5] B. Giffler, "Mathematical solution of production planning and scheduling problems", IBM ASDD Tech. Rept. (1960). 
[6] M. Yoeli, "A note on a generalization of boolean matrix theory", American Mathematical Monthly 68 (1961) 552-557.

[7] R.A. Cuninghame-Green, "Describing industrial processes with interference and approximating their steady-state behaviour", Operational Research Quarterly 13 (1962) 95-100.

[8] R. Cruon and Ph. Hervé, "Quelques résultats rélatifs à une structure algébrique et à son application au problème central de l'ordonnancement", Revue Francaise de Recherche Opérationelle 9 (1965) 3-19.

[9] V. Peteanu, "An algebra of the optimal path in networks", Mathematica 9 (1967) 335-342.

[10] C. Benzaken, "Structures algébriques des cheminements", in: G. Biorci, ed., Network and switching theory (Academic Press, 1968) pp. 40-57.

[11] P. Robert and J. Ferland, "Généralisation de l'algorithme de Warshall, Revue Francaise d'Automatique, Informatique, Recherche Operationelle (Série Verte) 2 (1968) 71-85.

[12] I. Tomescu, "Sur l'algorithme matriciel de B. Roy", Revue Francaise d'Automatique, Informatique, Recherche Operationelle (Série Verte) 2 (1968) 87-91.

[13] B.A. Carré, "An algebra for network routing problems", Journal of the Institute of Mathematics and its Applications 7 (1971) 273-294.

[14] D.R. Shier, "A decomposition algorithm for optimality problems in tree-structured networks", Discrete Mathematics 6 (1973) 175-189.

[15] E. Minieka and D.R. Shier, "A note on an algebra for the $k$ best routes in a network", Journal of the Institute of Mathematics and its Applications 11 (1973) 145-149.

[16] M. Gondran, "Algèbre des chemins et algorithmes", NATO conference on combinatorial mathematics, Versailles, 1974.

[17] R.A. Cuninghame-Green, "Minimax algebra I: Bands and belts", Memorandum no. 70, Department of Applied Mathematics, Technische Hogeschool, Twente, the Netherlands, 1975.

\section{Other references}

The reference list in Section 8 (which is certainly incomplete) has been restricted to publications in which algebraic structures similar to those discussed in Section 1, are explicitly used. Much relevant material is also contained in publications in the fields of game theory, graphs and networks, transportation, automata theory, theory of algorithms, discrete dynamic programming, machine scheduling, approximation theory, nonlinear optimisation and duality, convex programming and so on.

The inspiration for some of the earlier results in the field can be directly traced to the work of Lunc and others in relation to switching theory and boolean matrices - for references, see [6 and 24]. Related also is the work of Rockafeller [28] on bifunctions and convex processes. The following references give access to some of the wider issues.

[18] G. Birkhoff, Lattice theory (Am. Math. Soc., Providence, R.I., 1967).

[19] T.S. Blyth and M.F. Janowitz, Residuation theory (Pergamon, Oxford, 1972).

[20] R.G. Busacker and T.L. Saaty, Finite graphs and networks (McGraw Hill, New York, 1965). 
[21] E.W. Cheney, Introduction to approximation theory (McGraw Hill, New York, 1966).

[22] J.E. Falk and R.M. Soland, "An algorithm for separable nonconvex programming problems", Management Science A 15 (1974) 550-569.

[23] L. Fuchs, Partially ordered algebraic systems (Pergamon, Oxford, 1963).

[24] P.L. Hammer and S. Rudeanu, Boolean methods in operations research (Springer, Berlin, 1968).

[25] N.H. McCoy, The theory of rings (MacMillan, New York, 1964).

[26] L. McLinden, “An extension of Fenchel's duality theorem to saddle functions and dual minimax problems", Pacific Journal of Mathematics 50 (1974) 135-158.

[27] A.H.G. Rinnooy Kan, "The machine scheduling problem", Mathematics Centre, Amsterdam (1973).

[28] R.T. Rockafeller, Convex analysis (Princeton University Press, Princeton, N.J., 1970). 